\title{
Magnetohydrodynamic Origin of Jets from Accretion Disks
}

\author{
R.V.E. Lovelace, \\ Department of Astronomy, Cornell University, Ithaca, NY 14853; \\ rvl1@cornell.edu \\ G.V. Ustyugova \\ Keldysh Institute of Applied Mathematics, Russian Academy of \\ Sciences, Moscow, Russia, 125047, ustyugg@spp.Keldysh.ru \\ A.V. Koldoba \\ Institute of Mathematical Modelling, Russian Academy of Sciences, \\ Moscow, Russia, 125047
}

\begin{abstract}
A review is made of recent magnetohydrodynamic (MHD) theory and simulations of origin of jets from accretion disks. Many compact astrophysical objects emit powerful, highly-collimated, oppositely directed jets. Included are the extra galactic radio jets of active galaxies and quasars, and old compact stars in binaries, and emission line jets in young stellar objects. It is widely thought that these different jets arise from rotating, conducting accretion disks threaded by an ordered magnetic field. The twisting of the $\mathbf{B}$ field by the rotation of the disk drives the jets by magnetically extracting matter, angular momentum, and energy from the accretion disk. Two main regimes have been discussed theoretically, hydromagnetic winds which have a significant mass flux, and Poynting flux jets where the mass flux is negligible. Over the past several years, exciting new developments on models of jets have come from progress in MHD simulations which now allow the study of the origin the acceleration and collimation - of jets from accretion disks. Simulation studies in the hydromagnetic wind regime indicate that the outflows are accelerated close to their region of origin whereas the collimation occurs at much larger distances.
\end{abstract}

\section{Introduction}

Powerful, highly-collimated, oppositely directed jets are observed in active galaxies and quasars (see for example Bridle \& Eilek 1984), old compact stars in binaries (Mirabel \& Rodriguez 1994), and emission line jets in young stellar objects (Mundt 1985; Bührke, Mundt, \& Ray 1988). A broad spectrum of ideas and models have been put forward to explain astrophysical jets (see reviews by Begelman, Blandford, \& Rees 1984 and Bisnovatyi-Kogan 1993). The matter is thought to go to the jet from the inner region of an accretion disk surrounding 


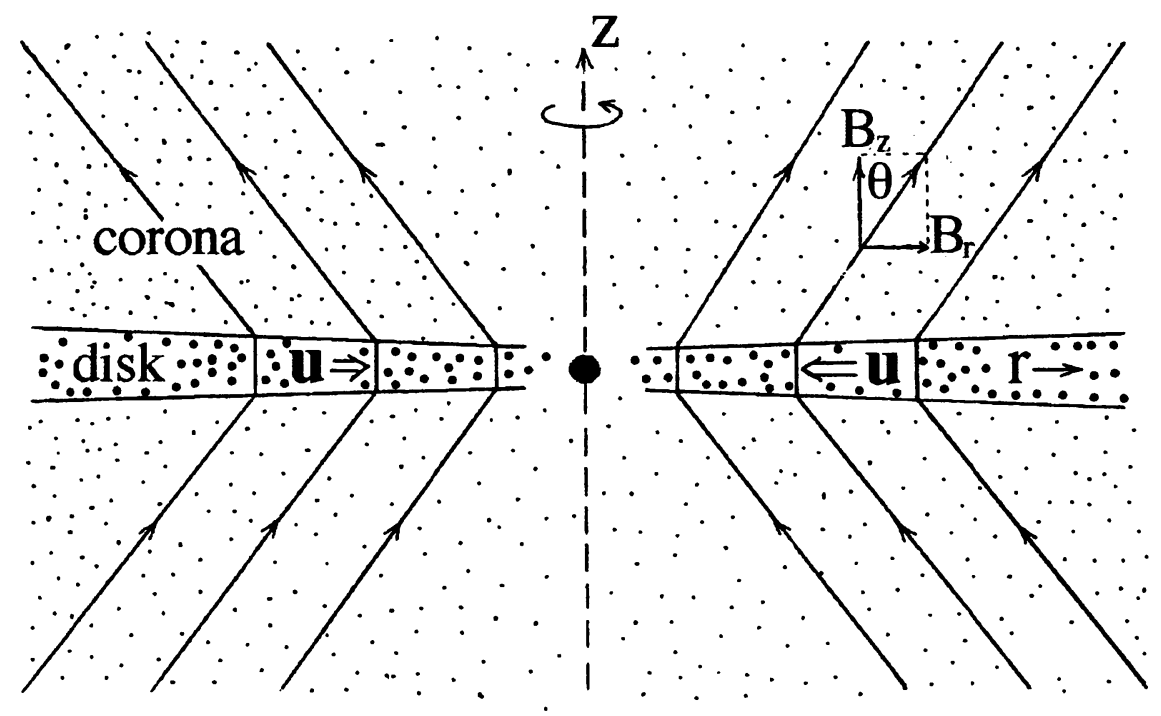

Figure 1. Sketch of an accretion disk threaded by a magnetic field for conditions which may lead to hydromagnetic jet formation.

the compact object - a star or black hole. The disk matter must then be accelerated to a velocity higher that the escape velocity from the central object. Further, the jet matter should have sufficient momentum to propagate through surrounding inter-stellar or intra-galactic matter out to large distances.

An ordered magnetic field is widely thought to have an essential role in jet formation from a rotating accretion disk. Two main regimes have been considered in theoretical models, the Poynting flux regime where the energy outflow from the disk is carried mainly by the electromagnetic field with the energy carried by the matter small, and the hydromagnetic regime where the energy is carried by both the electromagnetic field and the kinetic flux of matter. Poynting flux models for the origin of jets were proposed by Lovelace (1976) and Blandford (1976) and studied further by Lovelace et al. (1987) and Colgate \& $\mathrm{Li}$ (1998). In these models the rotation of a Keplerian accretion disk twists a poloidal field threading the disk, and this results in outflows out of the disk which carry angular momentum (in the twist of the field) and energy (in the Poynting flux) away from the disk, thereby facilitating the accretion of matter.

Important questions to be answered by the jet models include the following: 1. What is the main driving force pushing matter into the jet? $\mathbf{2}$. What determines the mass flow rate in the jet $\dot{M}_{j}$, and what fraction is this of the accretion rate? 3. What physics determines the asymptotic speed or Lorentz factor of the bulk flow? 4. What determines the collimation of the jet and at what distance from the central object does the jet become collimated? 5 . What is the acceleration mechanism of leptons to Lorentz factors $\gamma \sim 10^{2}-10^{3}$ in the radio jets? Observations of Blazars indicate that $\gamma \sim 10^{3}-10^{5}$ in some objects. 
The focus of recent work has been the hydromagnetic regime of jet formation for the geometry sketched in Figure 1. A strong case for hydromagnetic jets as an explanation of jets in protostellar systems emerges because the temperature of the inner regions of these systems is insufficient to permit driving by thermal or radiation pressure (Königl \& Ruden 1993). Part of the investigations have been analytical or semi-analytical and outgrowths of the self-similar solution of Blandford \& Payne (1982) (Pudritz \& Norman 1986; Königl 1989; Pelletier \& Pudritz 1992; Contopoulos \& Lovelace 1994). The outflows in this model are often referred to as "centrifugally driven" owing to the driving force close to the disk: If the poloidal magnetic field lines diverge from the disk surface (making an angle with the $z$-axis of more than $30^{\circ}$ ), then the sum of the gravitational and centrifugal forces is in the $+z$ direction for an MHD fluid particle which initially tends to maintain a constant angular rotation rate. The self-similar models are unsatisfactory in the respect that they must be cutoff at small cylindrical radii, $r \leq r_{\min }$. This is the most important region of the jet flow. Observations of optical stellar jets (Mundt 1985) reveal jet velocities $\sim 200-400 \mathrm{~km} / \mathrm{s}$, which are comparable to the Keplerian disk velocity close to the star's surface. This suggests that the jets originate from the inner region of the disk close to the star (Shu et al. 1988; Pringle 1989).

The limitations of analytical models has motivated efforts to study jet formation using MHD simulations. Simulation studies of hydromagnetic jet formation have addressed two main regions: The jet formation region where the matter enters with sub slow-magnetosonic speed and exits with super fast-magnetosonic speed. The second region includes the disk and the problem of the Velikhov (1959) - Chandrasekhar (1981) - Balbus-Hawley (1998) instability and the resulting 3D MHD turbulence. A number of studies have addressed the coupled problem of the disk and near jet regions (Uchida \& Shibata 1985; Shibata \& Uchida 1986; Stone \& Norman 1994; Bell and Lucek 1995;). MHD simulations of the near jet region have been carried out by several groups (Bell 1994; Ustyugova et al. 1995, 1998; Koldoba et al. 1995; Romanova et al. 1997, 1998; Meier et al. 1997; Ouyed \& Pudritz 1997).

Here, we first review recent results on MHD simulations of hydromagnetic jet formation and later discuss the Poynting flux regime. Section 2 discusses general considerations of hydromagnetic outflows. Sections 3 discusses MHD simulations which give non-stationary and stationary hydromagnetic outflows. Sections 4 describes the Poynting flux regime which remains to be fully explored by simulations. Section 5 gives the conclusions.

\section{Basics of MHD Outflows from Disks}

The main forces which drive a hydromagnetic outflow from a disk threaded by a magnetic field are the centrifugal force and the magnetic pressure gradient force. If disk has a hot corona, the pressure gradient may also be important. We neglect the radiative force but in this regard see Phinney (1987). Accreting matter of the disk carries magnetic field inward thus generating a $B_{r}$ component of the magnetic field as sketched in Figure 1. On the other hand, rotation of the disk acts to generate a toroidal component of the field $B_{\phi}\left(<0\right.$ if $\left.B_{z}>0\right)$. 
For a sufficiently inclined magnetic field ( $\theta$ in Figure 1 sufficiently large), outflows can result from the centrifugal force (Blandford \& Payne 1982) and/or the magnetic pressure gradient force $\left(-\nabla_{z} B_{\phi}^{2} /(8 \pi)\right)$ (Lovelace et al. 1989, 1991; Koupelis \& Van Horn 1989). This depends on the ratio of energy densities at the base of the outflow at the inner radius of the disk denoted $r_{i}$. Thus, the main parameters are $\varepsilon_{t h}=\left(c_{s} / v_{K}\right)_{i}^{2}$ and $\varepsilon_{B}=\left(v_{A} / v_{K}\right)_{i}^{2}$, where $v_{K}$ is Keplerian velocity, $c_{s}$ the sound speed, $v_{A}$ the Alfvén speed, and the $i$ subscript indicates evaluation on the surface of the disk at its inner radius, $r=r_{i}$. For $\varepsilon_{B} \sim 1$ the outflow is magnetically driven, whereas for $\varepsilon_{t h} \sim 1$, the flow is thermally driven.

Processes in the disk are of course coupled to the outflows (Lovelace et al. 1994, 1997; Falcke, Malkan, \& Biermann 1995). However, it is difficult to simulate both regions simultaneously because the time scales of the accretion and outflow are in general very different. The accretion is much slower. On the other hand the processes in the disk may involve the small scale MHD instability of Chandrasekhar, Velikhov, Balbus, and Hawley, and therefore require high spatial resolution. Stone \& Norman (1994) attempted to simultaneously simulate the internal MHD dynamics of a disk and MHD dynamics of outflows. This proved impractical because essentially all of the spatial resolution was needed for treating the unstable dynamics of the disk. Also, there was the problem that the initial configuration was far from equilibium. Simulation of the internal MHD disk dynamics has led several groups to the problem of simulating $3 \mathrm{D}$ MHD turbulence in a sheared flow of a patch of a disk (for example, Hawley et al. 1995; Brandenburg et al. 1995). This is a much larger project than that of understanding MHD outflows. At the same time it is widely thought, and observations of cataclysmic variables support the view, that the disk turbulence - including MHD turbulence - can be modeled approximately using the Shakura (1973), Shakura \& Sunyaev (1973) "alpha" viscosity model (Eardley \& Lightman 1975; Coroniti 1981). In contrast with the internal disk dynamics, there is theoretical and simulation evidence that the outflows can be treated using axisymmetric (2D) MHD (Blandford \& Payne 1982; Lovelace et al. 1991; Ustyugova et al. 1995). Here, we consider outflows from a disk represented as a boundary condition. This approach has subsequently been adopted by other groups (Meier et al. 1997; Ouyed \& Pudritz 1997). This treatment of the disk is justified for outflows from a disk where the accretion speed is small compared with the Keplerian speed (Ustyugova et al. 1995).

\section{Numerical Simulations of MHD Outflows}

In order to test the analytical models of stationary outflows, MHD simulations of flows from a disk treated as a boundary condition have been carried out by a number of groups.

\subsection{Non-Stationary Outflows}

The initial magnetic field configuration was chosen so that the magnetic field was significantly inclined to the disk $\left(\theta>30^{\circ}\right)$ over most of the disk surface (Ustyugova et al. 1995; Koldoba et al. 1995). The simulations involve solving the complete system of ideal non-relativistic MHD equations using a Godunovtype code assuming axisymmetry but taking into account all three components 

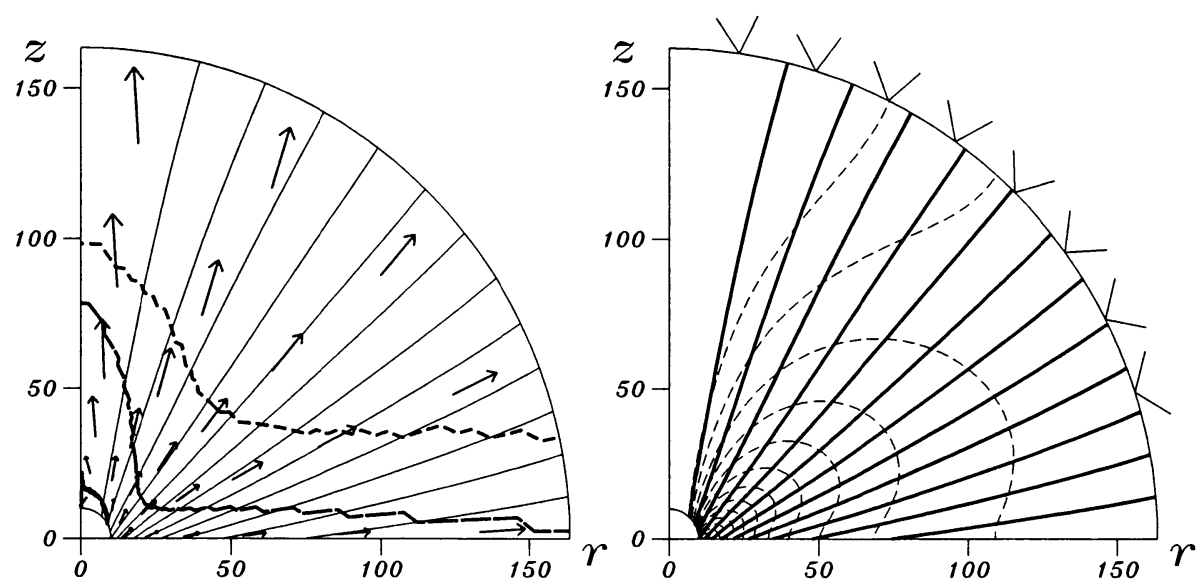

Figure 2. Simulations results for stationary MHD outflow obtained using a spherical coordinate system (Ustyugova et al. 1998). The solid lines represent the poloidal magnetic field, and the arrows the velocity vectors. The dashed lines in the left-hand plot represent the slow magnetosonic surface (the lowest dashed line), the Alfvén surface (the middle line), and the fast magnetosonic surface (the top line). In the right-hand panel, the dashed lines are surfaces of constant toroidal current density, while the lines on the outer boundary are the projections of the fast magnetosonic Mach cones.

of velocity and magnetic field. Matter of the corona was initially in thermal equilibrium with the gravitating center. At $t=0$, the disk is set into rotation with Keplerian velocity and at the same time matter is pushed from the disk with a small poloidal velocity equal to a fraction of the slow magnetosonic velocity $\left(v_{p}=\alpha v_{s m}\right.$, with $\left.\alpha=0.1-0.9\right)$. A relatively high temperature and small magnetic field was considered. We found that at the maximum of the outflow, matter is accelerated to speeds in excess of the escape speed and in excess of the fast magnetosonic speed within the simulation region $\left(\sim 100 r_{i}\right)$. The acceleration is due to both thermal and magnetic pressure gradients. The outflow collimates within the simulation region due to strong amplification, 'wrapping up' of the toroidal magnetic field and the associated pinching force.

However, the outflows are not stationary. The matter flux grows to a peak and then decreases to relatively small values. The strong collimation of the outflow reduces the divergence of the field away from the $z$-axis $\left(\theta<30^{\circ}\right)$ and this "turns off" the outflow of matter and leads to flow velocities less than the escape speed. Thus, this simulation is an example of a temporary outburst of matter to a jet. Unfortunately, this type of flow has a significant dependence on the initial conditions.

\subsection{Stationary Outflows}

More recently, stationary magnetohydrodynamic outflows from a rotating accretion disk have been obtained by time-dependent axisymmetric simulations by 


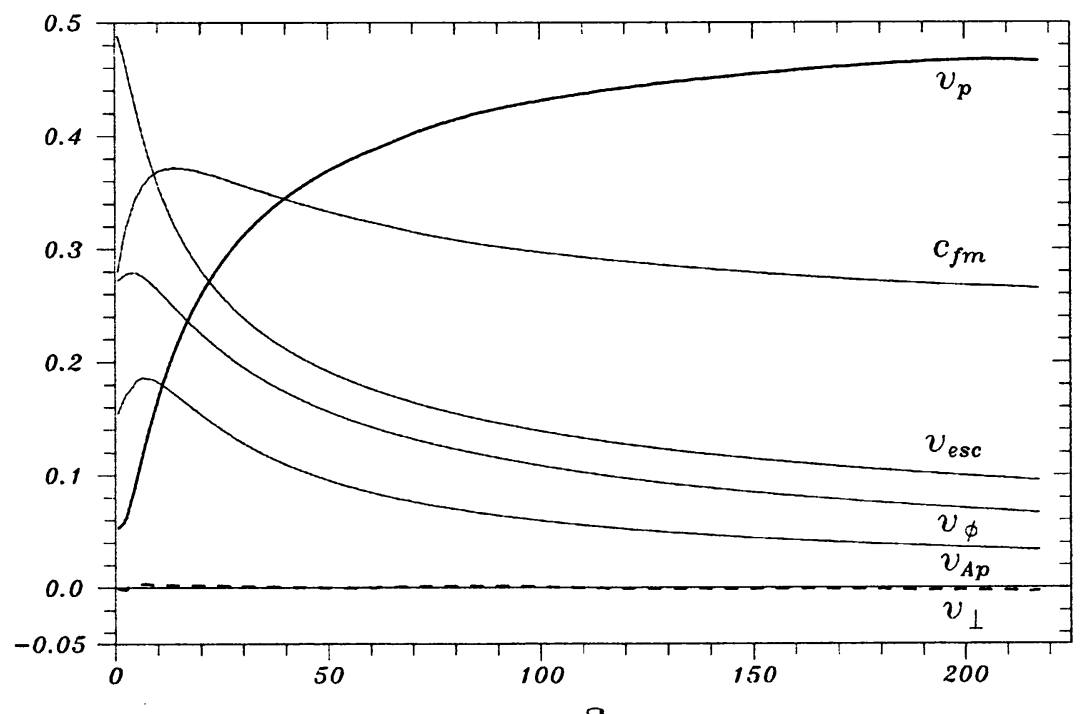

$S$

Figure 3. Dependences of different velocities on distance $s$ measured in units of $r_{i}$ from the disk along the second magnetic field line away from the axis in Figure 2 (Ustyugova et al. 1998). This field line "starts" from the disk at $r \approx 6 r_{i}$ where it has an angle $\theta \approx 28^{\circ}$ relative to the $z$-axis. The velocities are measured in units of $\sqrt{G M / r_{i}}$. Here, $v_{p}$ is the poloidal velocity along the field line and $v_{\perp}$ is the poloidal velocity perpendicular to the field line. Also, $v_{A p}$ is the poloidal Alfvén velocity, $c_{f m}$ is the fast magnetosonic velocity, and $v_{e s c}$ is the local escape velocity.

Romanova et al. (1997) and systematically analyzed by Ustyugova et al. (1998). The initial magnetic field in the latter work was taken to be a split-monopole poloidal field configuration (Sakurai 1987) frozen into the disk. The disk was treated as a perfectly conducting, time-independent density boundary $[\rho(r)]$ in Keplerian rotation which is different from our earlier specification of a small velocity outflow ( $\$ 3.1$, Ustyugova et al. 1995). The outflow velocity from the disk is determined self-consistently from the MHD equations. The temperature of the matter outflowing from the disk is small in the region where the magnetic field is inclined away from the symmetry axis $\left(c_{s}^{2} \ll v_{K}^{2}\right)$, but relatively high $\left(c_{s}^{2} \lesssim v_{K}^{2}\right)$ at very small radii in the disk where the magnetic field is not inclined away from the axis. We have found a large class of stationary MHD winds. Within the simulation region, the outflow accelerates from thermal velocity $\left(\sim c_{s}\right)$ to a much larger asymptotic poloidal flow velocity of the order of $0.5 \sqrt{G M / r_{i}}$, where $M$ is the mass of the central object and $r_{i}$ is the inner radius 
of the disk. This asymptotic velocity is much larger than the local escape speed and is larger than fast magnetosonic speed by a factor of $\sim 1.75$. The acceleration distance for the outflow, over which the flow accelerates from $\sim 0$ to, say, $90 \%$ of the asymptotic speed, occurs at a flow distance of about $80 r_{i}$. The flows are approximately spherical outflows, with only small collimation within the simulation region. The collimation distance over which the flow becomes collimated (with divergence less than, say, $10^{\circ}$ ) is much larger than the size of our simulation region. Close to the disk the outflow is driven by the centrifugal force while at all larger distances the flow is driven by the magnetic force which is proportional to $-\nabla\left(r B_{\phi}\right)^{2}$, where $B_{\phi}$ is the toroidal field.

The stationary MHD flow solutions allow us (1) to compare the results with MHD theory of stationary flows, (2) to investigate the influence of different outer boundary conditions on the flows, and (3) to investigate the influence of the shape of the simulation region on the flows. The ideal MHD integrals of motion (constants on flux surfaces discussed by Lovelace et al. 1986) were calculated along magnetic field lines and were shown to be constants with accuracy $5-15 \%$. Other characteristics of the numerical solutions were compared with the theory, including conditions at the Alfvén surface.

Different outer boundary conditions on the toroidal component of the magnetic field can significantly influence the calculated flows. The commonly used "free" boundary condition on the toroidal field leads to artificial magnetic forces on the outer boundaries, which can give spurious collimation of the flows. New outer boundary conditions which do not give artificial forces have been proposed and investigated by Ustyugova et al. (1998).

The simulated flows may also depend on the shape of the simulation region. Namely, if the simulation region is elongated in the $z$-direction, then Mach cones on the outer cylindrical boundaries may be partially directed inside the simulation region. Because of this, the boundary can have an artificial influence on the calculated flow. This effect is reduced if the computational region is approximately square or if it is spherical as in Figure 2. Simulations of MHD outflows with an elongated computational region can lead to artificial collimation of the flow.

Recent simulation studies have treated MHD outflows from disks with more general initial $\mathbf{B}$ field configurations, for example, that where the poloidal field has different polarities as a function of radius (Hayashi, Shibata, \& Matsumoto 1996; Goodson, Winglee, \& Böhm 1997; Romanova et al. 1998). The differential rotation of the foot-points of $\mathbf{B}$ field loops at different radii on the disk surface causes twisting of the coronal magnetic field, an increase in the coronal magnetic energy, and an opening of the loops in the region where the magnetic pressure is larger than the matter pressure $(\beta \lesssim 1)$ (Romanova et al. 1998). In the region where $\beta \gtrsim 1$, the loops may be only partially opened. Current layers form in the narrow regions which separate oppositely directed magnetic field. Reconnection occurs in these layers as a result of the small numerical magnetic diffusivity. In contrast with the case of the solar corona, there can be a steady outflow of energy and matter from the disk surface. We find that the power output mainly in the form of a Poynting flux. Opening of magnetic field loops and subsequent closing can give reconnection events which may be responsible for X-ray flares 
in disks around both stellar mass objects and massive black holes (Hayashi et al. 1996; Goodson, Winglee, \& Böhm, 1997; Romanova et al. 1998).

\section{Poynting Flux Jets}

In a very different regime from the hydromagnetic flows discussed in $\S 3$, a Poynting flux jet transports energy and angular momentum mainly by the electromagnetic fields with only small contributions of the matter (Lovelace et al. 1987; Colgate and Li 1998). A steady Poynting jet - sketched in Figure 4 - can be characterized in the lab frame by its asymptotic (large distance) magnetic field $B_{\phi}=-B_{0}\left[r_{o} / r_{j}(z)\right]$ at the jet's edge, $r=r_{j}(z)$, where $r_{0}$ is the jet's radius at $z=0$ and $B_{0}$ is the poloidal field strength at this location. The electric

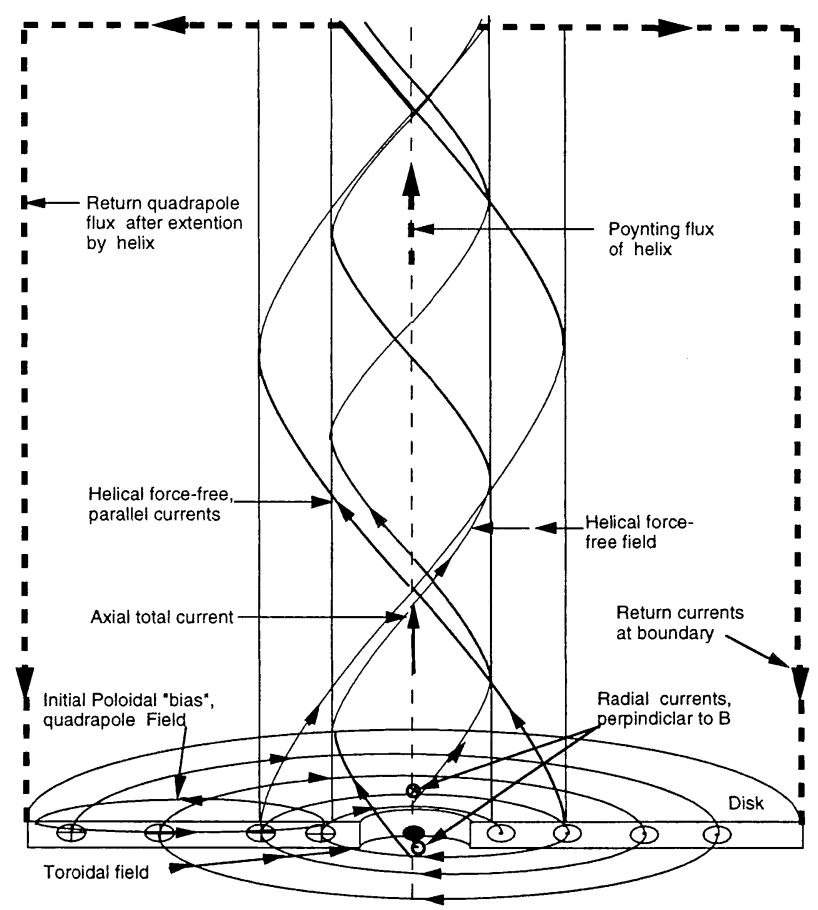

Figure 4. Field configuration of a Poynting flux jet from Colgate and $\operatorname{Li}(1998)$.

field in the jet is $\mathbf{E}=-\mathbf{v} \times \mathbf{B} / \boldsymbol{c}$ and consequently the energy flux or luminosity of the jet is $L_{j}=v B_{0}^{2} r_{0}^{2} / 8 \sim 4 \times 10^{45} \mathrm{erg} / \mathrm{s}(v / c)\left(r_{0} / 10^{14} \mathrm{~cm}\right)^{2}\left(B_{0} / 10^{4} \mathrm{G}\right)^{2}$. Propagating disturbances in such field dominated jets provide a simple, but 
self-consistent physical model for the gamma ray flares observed in Blazars (Romanova \& Lovelace 1997; Levinson 1998; Romanova these proceedings). Owing to pair production close to the black hole, the main constituent of a Poynting flux jet may be electron-positron pairs.

\section{Conclusions}

MHD simulations carried out by a number of groups over the last several years support the idea that an ordered magnetic field of an accretion disk can give powerful outflows of matter, energy, and angular momentum. The studies so far have been in the hydromagnetic regime and find asymptotic flow speeds of the order of the maximum Keplerian velocity of the disk. In contrast, observed VLBI jets in quasars and active galaxies point to bulk Lorentz factors of order 10 - much larger than the disk Lorentz factor. This may be a result of the relativistic dynamics not included here, but more likely it reflects the fact that these jets are in the Poynting flux regime. Also, these jets may involve energy extraction from a rotating black hole (Blandford and Znajek 1977; Livio et al. 1998).

Acknowledgments. It is a pleasure to thank the organizers for a most memorable meeting. This work was supported in part by NSF grant AST9320068 and NASA grant NAG5 6311. The Russian authors were supported in part by RFFI Grant 96-02-17113. Also, the research described here was made possible in part by Grant No. RP1-173 of the U.S. Civilian R\&D Foundation for the Independent States of the Former Soviet Union.

\section{References}

Balbus, S.A., \& Hawley, J.F. 1998, Rev. Mod. Phys., 70, 1

Bisnovatyi-Kogan, G.S. 1993, in Stellar Jets and Bipolar Outflows, L. Errico \& A.A. Vittone, eds. Dordrecht: Kluwer, 369

Begelman, M.C., Blandford, R.D., \& Rees, M.J. 1984, Rev. Mod. Phys., 56, 255

Bell, A.R. 1994. Phys. Plasmas, 1, 1643

Bell, A.R., \& Lucek, S.G. 1995, MNRAS, 277, 1327

Blandford, R.D. 1976, MNRAS, 1976, 465

Blandford, R.D., \& Payne, D.G. 1982, MNRAS, 199, 883

Blandford, R.D., \& Znajek, R.L. 1977, MNRAS, 179, 433

Brandenburg, A., Nordlund, A., Stein, R.F., \& Torkelson, U. 1995, ApJ, 446, 741

Bridle, A.H., \& Eilek, J.A. (eds) 1984, in Physics of Energy Transport in Extragalactic Radio Sources, Greenbank: NRAO

Bührke, T., Mundt, R., \& Ray, T.P. 1988, A\&A, 99

Chandrasekhar, S. 1981, Hydrodynamic and Hydromagnetic Stability, (New York: Dover) 
Colgate, S.A. \& Li, H. 1998, in Proc. of VII International Conference and Lindau Workshop on Plasma Astrophysics and Space Physics, Lindau, Germany

Contopoulos, J., \& Lovelace, R.V.E. 1994, ApJ, 429, 139

Coroniti, E.V. 1981, ApJ, 244, 587

Eardley, D.M. \& Lightman, A.P. 1975. ApJ, 200, 187

Falcke, H., Malkan, M.A., \& Biermann, P.L. 1995, A\&A, 298, 375

Goodson, A.P., Winglee, R.M., \& Böhm, K.H. 1997, ApJ, 489, 390

Hawley, J.F., Gammie, C.F., \& Balbus, S.A. 1995, ApJ, 440, 742

Hayashi, M.R., Shibata, K., \& Matsumoto, R. 1996, ApJ, 468, L37

Koldoba, A.V., Ustyugova, G.V., Romanova, M.M., Chechetkin, V.M., \& Lovelace, R.V.E. 1995, Ap\&SS, 232, 241

Königl, A. 1989, ApJ, 342, 208

Königl, A., \& Ruden, S.P. 1993, Protostars and Planets III, E.H. Levy and J. Lunine, Tucson: Univ. of Arizona Press, 641

Koupelis, T., \& Van Horn, H.M. 1989, ApJ, 342, 146

Levinson, A. 1998, ApJ, 507, 145

Livio, M., Ogilvie, G.I., \& Pringle, J.E. 1998, ApJ, submitted

Lovelace, R.V.E. 1976, Nature, 262, 649

Lovelace, R.V.E., Mehanian, C., Mobarry, C.M., \& Sulkanen, M.E. 1986, ApJ Suppl., 62,1

Lovelace, R.V.E., Wang, J.C.L., \& Sulkanen, M.E. 1987, ApJ, 315, 504

Lovelace, R.V.E., Mobarry, C.M., \& Contopoulos, J. 1989, in Accretion Disks and Magnetic Fields in Astrophysics, ed. G. Belvedere (Dordrecht: Kluwer), 71

Lovelace, R.V.E., Berk, H.L., \& Contopoulos, J. 1991, ApJ, 379, 696

Lovelace, R.V.E., Romanova, M.M.,\& Contopoulos, J. 1993, ApJ, 403, 158

Lovelace, R.V.E., Romanova, M.M., \& Newman, W.I. 1994, ApJ, 437, 136

Lovelace, R.V.E., Newman, W.I., \& Romanova, M.M., 1997, ApJ, 424, 628

Meier, D.L., Edgington, S., Godon, P., Payne, D.G., \& Lind, K.R. 1997, Nature, 388,350

Mirabel, I.F., \& Rodriguez, L.F. 1994 Nature, 371, 46

Mundt, R. 1985, in Protostars and Planets II, D.C. Black and M.S. Mathews, eds. Univ. of Arizona Press, Tucson, 414

Oyed, R. \& Pudritz, R.E. 1997, ApJ, 482, 712

Pelletier, G., \& Pudritz, R.E. 1992, ApJ, 394, 117

Phinney, E.S. 1987, in Superluminal Radio Sources, J.A. Zensus, \& T.J. Pearson, Cambridge: Cambridge Univ. Press, 301

Pringle, J.E., 1989, MNRAS, 236, 107

Pudritz, R.E., \& Norman, C.A. 1986, ApJ, 301, 571

Romanova, M.M., \& Lovelace, R.V.E. 1997, ApJ, 475, 97

Romanova, M.M., Ustyugova, G.V., Koldoba, A.V., Chechetkin, V.M., \& Lovelace, R.V.E. 1997, ApJ, 482, 708 
Romanova, M.M., Ustyugova, G.V., Koldoba, A.V., Chechetkin, V.M., \& Lovelace, R.V.E. 1998, ApJ, 500, 703

Sakurai, T. 1987, PASJ, 39, 821

Shakura, N.I. 1973, Soviet Astron., 15, 377

Shakura, N.I., \& Sunyaev, R.A. 1973, A\&A, 24, 337

Shibata, K., \& Uchida, Y. 1986, PASJ, 38, 631

Shu, F.H., Lizano, S., Ruden, S.P., \& Najita, J. 1988, ApJ, 328, L19

Stone, J.M., \& Norman, M.L. 1994, ApJ, 433, 746

Uchida,Y, \& Shibata, K. 1985, PASJ, 37, 515

Ustyugova, G.V., Koldoba, A.V., Romanova, M.M., Chechetkin, V.M., \& Lovelace, R.V.E. 1995, ApJ, 439, L39

Ustyugova, G.V., Koldoba, A.V., Romanova, M.M., Chechetkin, V.M., \& Lovelace, R.V.E. 1998, ApJ, in press (astro-ph/9812284)

Velikhov, E.P. 1959, J. Exp. Theo. Phys., 36, 1398 